\title{
Daily stressors and negative life events in children at elevated risk of developing schizophrenia
}

\author{
Alexis E. Cullen, Helen L. Fisher, Ruth E. Roberts, Carmine M. Pariante and Kristin R. Laurens*
}

\section{Background}

Psychological stress is implicated in the development of schizophrenia, but little is known about experiences of stress among children at elevated risk for the disorder.

\begin{abstract}
Aims
To examine stressor exposure and reactivity in children with different vulnerability profiles for schizophrenia: (a) children presenting multiple antecedents of schizophrenia (ASz group), (b) children with a family history of schizophrenia (FHx group) and (C) typically developing low-risk (TD) children.
\end{abstract}

\section{Method}

Ninety-five children ( $A S Z=29 ; F H x=19 ; A S Z+F H x=5$; $T D=42$ ), identified aged 9-12 years using a community-based screening procedure or as relatives of individuals with schizophrenia, completed questionnaires assessing environmental stressors and psychopathology at age 11-14 years.

\section{Results}

Relative to their typically developing peers, children in the $\mathrm{FHX}$ and ASz groups were exposed to a greater number of negative life events and a higher frequency of daily stressors, respectively; and were more distressed by these experiences.

\section{Conclusions}

Stress exposure and reactivity may constitute useful targets of early intervention for psychosis.

\section{Declaration of interest}

None.
The diathesis-stress model of schizophrenia proposes that environmental stressors interact with biological vulnerability to give rise to psychosis. ${ }^{1}$ Indeed, retrospective studies of patients with schizophrenia and prospective population cohort studies indicate that environmental stressors (encompassing major life events and childhood trauma, as well as milder daily stressors/ hassles) contribute to the development and maintenance of psychosis. $^{2-4}$ However, few studies have examined experiences of stress prospectively among children at elevated risk of developing the disorder in later life. Such studies are advantageous as recall bias is minimised; furthermore, elucidating causal pathways in children at elevated risk provides the exciting prospect of preventative intervention. Subclinical psychotic symptoms reported at interview have been used to distinguish children who may be at elevated risk for schizophrenia. ${ }^{5}$ Kelleher and colleagues examined trauma exposure in this population and found that, relative to healthy children without these symptoms, those putatively at greater risk were more likely to have experienced physical abuse and exposure to domestic violence. ${ }^{6}$ Furthermore, a study of adolescents at elevated risk of developing schizophrenia on account of their diagnosis of schizotypal personality disorder reported that these youth experienced more major life events than their healthy peers and greater distress resulting from daily stressors. ${ }^{7}$ Our group is the first in the UK to prospectively investigate how children at elevated risk for schizophrenia are affected by environmental stress. We examined experiences of negative life events and daily stressors among children with different vulnerability profiles for psychosis, including: (a) children at putatively greater risk who present multiple antecedents of schizophrenia (ASz group), ${ }^{8,9}$ (b) high-risk children with a family history of schizophrenia (FHx group), and (c) typically developing low-risk children (TD group). We hypothesised that children at elevated risk for schizophrenia would experience greater exposure to

*Senior author environmental stressors than their low-risk peers and greater distress in relation to these experiences. We also examined the extent to which environmental stressors were associated with current psychopathology.

\section{Method}

\section{Sampling and recruitment}

Traditionally, identification of individuals at elevated risk for developing schizophrenia has focused on those with a family history of illness. However, as the majority of individuals who develop schizophrenia do not have a relative with the disorder, ${ }^{10,11}$ alternative identification strategies have emerged. ${ }^{5,8,12}$ Our group has developed a novel, cost-effective and feasible communityscreening method that uses questionnaires to identify children who present a triad of well-replicated antecedents of schizophrenia, including: (a) speech and/or motor developmental delays or abnormalities; (b) social, emotional and/or behavioural problems; and (c) psychotic-like experiences (subclinical psychotic symptoms). ${ }^{8,9}$ Although only longitudinal follow-up can determine the specificity and sensitivity of the triad in predicting later schizophrenia, preliminary evidence demonstrates that, relative to their typically developing peers, these children present several features that characterise adults with schizophrenia, including functional brain abnormality following commission of behavioural errors, ${ }^{13}$ involuntary dyskinetic movement abnormalities, ${ }^{14}$ structural brain abnormalities encompassing the temporal lobe, ${ }^{15}$ poorer intellectual and cognitive functioning, ${ }^{16}$ elevated social withdrawal ${ }^{17}$ and impairments in facial emotion recognition. ${ }^{18}$

The antecedent screening procedure was conducted in London primary schools. ${ }^{8,19}$ Children aged 9-12 years completed questionnaires at school and caregivers completed questionnaires at home. Delays or abnormalities in speech and/or motor development were assessed via caregiver-report using three quantitative and six qualitative questions, which demarcated gross 
deviations in milestone attainment and professional and parental concerns regarding speech/motor development. ${ }^{8}$ Social, emotional and behavioural problems were defined as a score in the clinical range (approximating the top tenth percentile on UK population norms) on at least one of the four Strengths and Difficulties Questionnaire (SDQ) ${ }^{20}$ psychopathology scales, including child-reported emotional symptoms, or caregiverreported conduct problems, hyperactivity-inattention, or peer relationship problems. The presence of a psychotic-like experience was defined as a child-reported 'certainly-true' response on at least one psychotic-like experience among nine items (described later).

To identify children with a family history of schizophrenia or schizoaffective disorder, the caregiver questionnaire also assessed family mental health difficulties. Additionally, medical records of mental health service users within the South London and Maudsley (SLaM) National Health Service (NHS) Foundation Trust were reviewed to identify patients with a diagnosis of schizophrenia or schizoaffective disorder who had a relative aged between 9 and 12 years. Identified families were approached following liaison with the patient's care worker.

Participants in our FHx group (identified either via community screening or medical records) had at least one firstor second-degree relative with schizophrenia or schizoaffective disorder, as confirmed by the Family Interview for Genetic Studies (FIGS). ${ }^{21}$ Participants in our ASz group were those who presented with each of the three antecedents assessed using the screening questionnaire. The TD group comprised children who presented none of the three antecedents on screening questionnaires and who had no first-, second- or third-degree relative with a schizophrenia spectrum disorder (confirmed via the FIGS). Exclusion criteria for all groups were insufficient English-language ability to complete assessments and a neurological condition that affected developmental milestone attainment or current functioning (e.g. epilepsy, cerebral palsy).

Screening questionnaires were completed by 1343 children and caregivers. Of these, 9.5\% $(n=128)$ of children presented with the triad of antecedents of schizophrenia, 22.5\% $(n=302)$ met criteria for the TD group. Of the 1020 children for whom family history information on screening questionnaires was available, $3.4 \%(n=35)$ were reported to have a family history of schizophrenia. A further 36 children who were eligible for the FHx group (i.e. who had a family history of schizophrenia, with viable contact details and fell within the appropriate age range) were identified via medical records. Eligible children meeting the criteria for each group were invited to participate in a longitudinal study of child development. In total, $41 \%(n=26)$ of the families of children with antecedents of schizophrenia and $42 \% \quad(n=36)$ of the families of typically developing children declined participation respectively, and $40 \%(n=17)$ of families with a history of schizophrenia identified either via the school screening procedure or via medical records declined to participate after initial contact. Children in the ASz and TD groups who participated in the study did not differ on age, gender or ethnicity from those who did not participate in these groups. For children with antecedents of schizophrenia, the prevalence of the triad components did not differ significantly between those who participated and those who did not, with the exception that SDQ emotional symptoms in the clinical range was less prevalent in those who participated than among those who did not.

\section{Procedure and measures}

This paper reports cross-sectional data from assessments completed when children were aged 11-14 years (i.e. on average,
32 months following their identification at age 9-12 years). Ethical permission for the study was granted by the Joint SLaM and Institute of Psychiatry NHS Research Ethics Committee. Caregivers and children provided written informed consent and assent, respectively, for participation.

\section{Demographic measures}

Caregivers current occupational status was elicited via semistructured interview and coded into three classes according to the UK National Statistics socioeconomic classification: ${ }^{22}$ (a) higher managerial, administrative and professional occupations; (b) intermediate occupations; and (c) routine and manual occupations. Participant ethnicity was determined using detailed information obtained from caregivers during the FIGS interview. $^{21}$

\section{Outcome measures}

\section{Negative life events}

An eight-item measure assessed exposure to a range of childappropriate negative life events. ${ }^{23}$ For each event, participants provided three ratings: (a) whether they had experienced the event (yes/no); (b) how distressed they felt at the time of the event; and (c) how much the event distressed them currently. Both distress ratings were scored on a four-point scale ( 0 , not at all; 1 , a little; 2 , somewhat; 3 , a lot). The number of negative life events was summed to provide a total score and two average distress scores (previous and current) were derived by dividing the sum of distress ratings by the total number of negative life events endorsed.

\section{Daily stressors}

Children completed a 37-item questionnaire, adapted from Heubeck \& O’Sullivan, ${ }^{23}$ assessing school-related daily stressors in four domains: scholastic ( 9 items: such as 'I have to sit a test'), home ( 4 items, such as 'My parents want me to do better'), peer ( 16 items, such as 'Other children make fun of me, tease me, or pick on me') and teacher ( 8 items, such as 'A teacher is unfair to me or other children'). For each item, participants indicated on a four-point scale how frequently the stressor had occurred during the past 6 months ( 0 , never; 1 , rarely; 2, sometimes; 3 , often), and how distressed this event made them feel ( 0 , not at all; 1, a little; 2, somewhat; 3, a lot). Frequency ratings for items included in each subscale were summed to give domain frequency scores (reflecting the number of stressors experienced and how often they occurred). A total daily stressor frequency score was computed by summing frequency scores on all items. Average domain distress scores were created by summing distress ratings on contributing items and dividing this score by the number of endorsed items (reflecting the average distress per item); the same procedure was performed with all items to obtain an overall average daily stressor distress score. After excluding one item from the scholastic domain, which was poorly correlated with the remaining items ('I have trouble with reading, writing, or spelling'), Cronbach's alpha coefficients were moderate to high (range: $0.71-$ 0.89 ) for all domain frequency and distress scales except the home frequency scale $(\alpha=0.55)$ and the home distress scale $(\alpha=0.59)$, which likely reflects the few items contributing to the home domain. Both scales were retained in analyses but should be interpreted with caution. Internal consistencies for the total frequency and overall distress scales were high $(0.88$ and 0.92 respectively; note, both scales included the item excluded from the scholastic domain). 


\section{Externalising and internalising psychopathology}

The Child Behaviour Checklist (CBCL; for children aged 6-18 years) ${ }^{24}$ and the corresponding Youth Self-Report (YSR; for children aged 11-18 years) were completed by the caregiver and the child respectively. These checklists are among the most widely used questionnaire measures of childhood psychopathology and exhibit high reliability and validity. ${ }^{24}$ They assess problems occurring during the past 6 months (CBCL, 113 items; YSR, 112 items), with each item scored on a three-point scale ( 0 , not true; 1 , somewhat true or sometimes true; 2 , very true or often true). The current study examined caregiver- and child-reported scores on the externalising and internalising scales.

\section{Psychotic-like experiences}

Children recompleted the nine-item psychotic-like experiences measure included in the antecedent screening questionnaire ${ }^{8,25}$ to provide ratings concurrently with the assessment of stressors. Five items were adapted from the Diagnostic Interview Schedule for Children ${ }^{26}$ (auditory hallucinations, thoughts read, ideas of reference, paranoid ideas and ideas of somatic changes), with four additional items assessing visual hallucinations, passivity phenomena, telepathic experiences and grandiosity. Each item was rated on a three-point scale ( 0 , not true; 1 , somewhat true; 2, certainly true) and summed to provide a total score. This measure exhibits sound psychometric properties, with all nine items loading onto a single latent psychotic-like construct. $^{25}$

\section{Statistical analyses}

Independent samples $t$-tests, Mann-Whitney $U$-tests, chi-squared tests and Fisher's exact tests were used to examine group differences on demographic variables. Linear regression analyses were employed to determine whether group status predicted the total number of negative life events, scores on the negative life event distress scales (previous and current), and the four daily stressor domain frequency and distress scales. As the two negative life event distress variables showed minor departures from normality, additional non-parametric analyses were conducted on these two variables using quantile (median) regression to confirm the pattern of results obtained in the parametric analyses (online Table DS1). In each analysis, the predictive effect of each risk group was tested independently (i.e. the ASz and FHx groups were examined relative to the TD group but were not directly compared with each other because of several children meeting inclusion criteria for both groups). All regression analyses were subsequently adjusted for demographic factors that differed significantly between the groups. To determine whether any effects of group status might be explained by current mood or distress relating to other psychopathology, analyses examining the effect of group status on distress relating to environmental stressors were additionally adjusted for child-reported current psychopathology (internalising and externalising symptoms and psychotic-like experiences). Unstandardised regression coefficients from both unadjusted and adjusted analyses were used to derive standardised mean differences $(d)$ as indices of effect size. ${ }^{27}$ Exploratory correlation analyses were next conducted to examine relationships between environmental stressors and current psychopathology. Non-parametric (Spearman's rho ' $\rho$ ') correlation analyses were performed as symptom scores were not normally distributed. To limit the number of statistical tests, only total scores on the daily stressor frequency and distress scales (as opposed to the four domain scores individually) were examined in the correlation analyses. Analyses were conducted using SPSS version 20 and Stata version 11 on Windows.

\section{Results}

\section{Sample characteristics}

In total, 95 children participated in the study; 42 met TD criteria, 29 met ASz criteria only, 19 met FHx criteria only, and 5 children met both $\mathrm{ASz}$ and FHx criteria. The five participants who met both $\mathrm{ASz}$ and FHx criteria were included in both the ASz and FHx groups, yielding data for 34 individuals in the ASz group and 24 individuals in the FHx group in total. As described previously, data from these non-mutually exclusive ASz and FHx groups were examined relative to the TD group only, and not compared directly with each other. Analyses were subsequently repeated for each group after excluding the five children meeting both $\mathrm{ASz}$ and FHx criteria.

Demographic characteristics are presented by group in Table 1 . Across the total sample, the average age at assessment was 13.1 years. There were no significant group differences in age or lapse of time between screening (recruitment) and assessment. Children in the ASz group were significantly more likely to be male compared with the TD group $(P=0.04)$. When $\mathrm{ASz}$ and $\mathrm{FHx}$ groups were compared with the TD group, they each differed significantly on ethnicity $(P<0.05)$ and socioeconomic status $(P<0.001)$. Relative to the TD group, the ASz group showed significantly higher scores on the CBCL internalising and externalising scales, the YSR externalising scale and the nine-item psychotic-like experience questionnaire $(P<0.05)$, and the FHx group obtained significantly higher scores on the YSR externalising scale $(P=0.05)$.

\section{Group differences in negative life events}

Prevalence data for each negative life event are presented by group in Table 2. The mean number of negative life events experienced by the ASz, FHx and TD groups was 1.74, 1.96 and 1.10 respectively (Table 3 ). In unadjusted analyses (Table 3 ), the FHx group reported a significantly higher number of negative life events than the TD group, equating to a moderate effect size $(d=0.66, P=0.01)$; this effect was largely unchanged $(d=0.77$, $P=0.02)$ after adjustment for demographic factors (i.e. gender, ethnicity and socioeconomic status). In unadjusted analyses, differences between the FHx and the TD groups on the level of distress experienced in relation to negative life events at the time of the event and currently were small-to-moderate in magnitude ( $d=0.22$ and $d=0.41$ respectively, $P>0.05)$. After adjustment for demographic factors and current psychopathology (YSR externalising and internalising symptoms and psychotic-like experiences), the effect of FHx status on both distress variables increased in magnitude and statistical significance (distress at the time: $d=0.62, P=0.08$; current distress: $d=0.90, P=0.01$ ). Children in the $\mathrm{ASz}$ group also reported a greater number of negative life events relative to the TD group $(d=0.55, P=0.02)$; an effect that was slightly attenuated after adjustment for demographic factors, but remaining of moderate magnitude $(d=0.48, P=0.09)$. In contrast, there were no significant differences between the ASz and TD groups on the degree of distress experienced either at the time of the event or currently in unadjusted or adjusted analyses $(d<0.37$ for all four comparisons, $P>0.05$ ). Quantile regression analyses performed on the negative life event distress variables showed an identical pattern of results (Table DS1).

After excluding the five children meeting both ASx and FHx criteria, all group differences were reduced in magnitude. The FHx group continued to report a higher number of negative life events than those in the TD group, although these differences reduced to a small-to-moderate magnitude in both adjusted and 


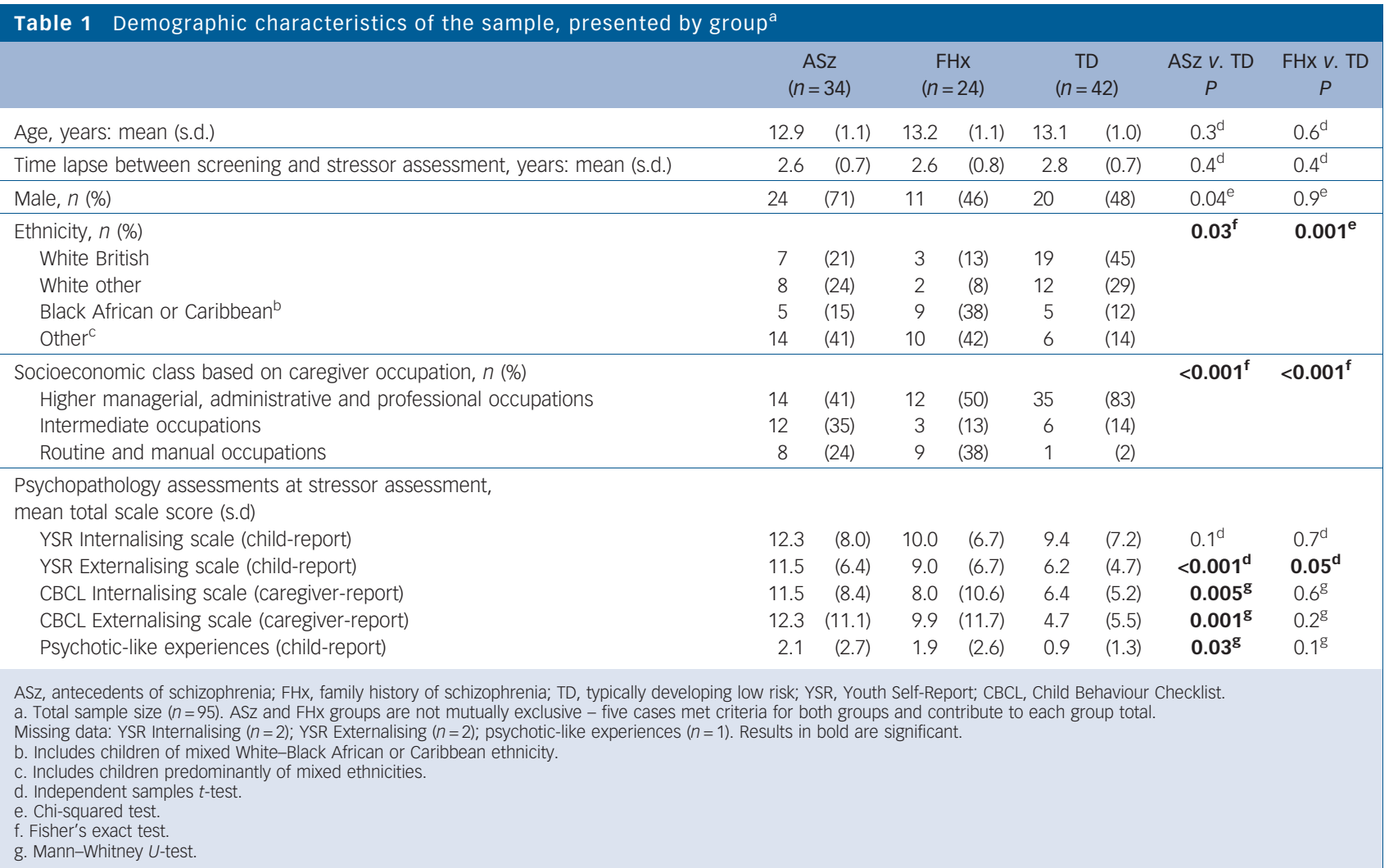

unadjusted analyses ( $d=0.43$ for both, $P>0.05$ ). The effect of FHx status on current distress relating to negative life events remained large in magnitude $(d=0.82, P=0.03)$. There were no differences between the $\mathrm{ASz}$ and TD groups on any variable.

\section{Group differences in daily stressors}

Table 3 presents the mean daily stressor frequency and distress scores by group and the results of the unadjusted linear regression analyses. Relative to the TD group, the ASz group were exposed to a higher frequency of stressors in the peer $(d=0.84, P=0.001)$ and teacher $(d=0.90, P<0.001)$ domains; effects remained moderateto-large after adjustment for demographic factors (peer: $d=1.02$, $P=0.001$; teacher: $d=0.73, P=0.008$ ). Children in the ASz group reported significantly greater distress regarding daily stressors across all four domains than the TD group $(d=0.53-0.63$, $P<0.03)$. After adjustment for demographic factors and current psychopathology, the magnitudes of effect were unchanged for scholastic-, peer- and teacher-related distress scales $(d=0.60-$ $0.62, P<0.05)$; however, the effect of ASz status on home-related distress was substantially reduced $(d=-0.01, P=0.98)$. In contrast, relative to the TD group, the FHx group reported that they were more frequently exposed to home-related stressors ( $d=0.53, P=0.05)$ and that they were more distressed by these experiences $(d=0.53, P=0.05)$. Both effects were reduced in magnitude in adjusted analyses $(d=0.24$ and 0.42 respectively, $P>0.05)$.

After excluding the five children meeting both ASz and FHx criteria, the $\mathrm{ASz}$ group continued to report a higher frequency of peer- and teacher-related daily stressors than the TD group $(d \geqslant 0.80, P<0.002)$, which remained unchanged after adjusting for demographic factors $(d \geqslant 0.72, P<0.01)$. The ASz group also experienced greater distress in the scholastic, peer and teacher domains $(d=0.49-0.55, P<0.05)$; the magnitude of all effects were attenuated after adjustment for demographic factors and current psychopathology with the exception of teacher-related distress $(d=0.63, P=0.05)$. There were no differences between the FHx and TD groups.

\section{Associations between symptoms, negative life events and daily stressors}

In the $\mathrm{ASz}$ group, psychotic-like experiences were strongly correlated with total scores on the daily stressor frequency scale ( $\rho=0.53, P=0.001)$ and with the number of negative life events

Table 2 Prevalence of negative life events, presented by group ${ }^{a}$

\begin{tabular}{|c|c|c|c|}
\hline & \multicolumn{3}{|c|}{$n(\%)$} \\
\hline & $\begin{array}{l}\text { ASz group } \\
(n=34)\end{array}$ & $\begin{array}{l}\text { FHx group } \\
\quad(n=24)\end{array}$ & $\begin{array}{l}\text { TD group } \\
(n=42)\end{array}$ \\
\hline $\begin{array}{l}\text { Reported at least one negative } \\
\text { life event }\end{array}$ & $30(88)$ & $21(88)$ & 27 (64) \\
\hline $\begin{array}{l}\text { Prevalence of individual events } \\
\text { Death of someone close } \\
\text { Parental separation/divorce } \\
\text { Exposure to fire or natural } \\
\text { disaster } \\
\text { Involved in serious car accident } \\
\text { Serious illness (child) requiring } \\
\text { hospitalisation } \\
\text { Parent experienced serious } \\
\text { accident or illness } \\
\text { Victim of burglary } \\
\text { Victim of other crime }\end{array}$ & $\begin{array}{l}16(47) \\
17(50) \\
3(9) \\
1(3) \\
8(25) \\
5(15) \\
3(9) \\
6(18)\end{array}$ & $\begin{array}{l}8(35) \\
4(17) \\
3(13) \\
4(17)\end{array}$ & $\begin{array}{l}2(5) \\
2(5) \\
6(14) \\
3(7) \\
9(22) \\
4(10)\end{array}$ \\
\hline $\begin{array}{l}\text { ASz group, antecedents of schizophrenia } \\
\text { schizophrenia group; TD group, typically } \\
\text { a. Total sample size ( } n=95 \text { ): the ASz and } \\
\text { five children met criteria for both groups } \\
\text { data for individual items: death of some } \\
\text { requiring hospitalisation ( } \mathrm{ASZ}=2, \mathrm{FHX}=1\end{array}$ & $\begin{array}{l}\text { Dup; FHx grou } \\
\text { eloping low-1 } \\
\text { x groups are } \\
\text { d contribute } \\
\text { close }(T D=3 \\
\text { ctim of burgl }\end{array}$ & $\begin{array}{l}\text { lp, family histor } \\
\text { isk group. } \\
\text { not mutually ex } \\
\text { :o each group tc } \\
\text {; serious illness } \\
\text { ary }(T D=1)\end{array}$ & $\begin{array}{l}\text { of } \\
\text { clusive - } \\
\text { tal. Missing } \\
\text { (child }\end{array}$ \\
\hline
\end{tabular}


Table 3 Frequency and impact of negative life events and daily stressors, presented by group ${ }^{a}$

\begin{tabular}{|c|c|c|c|c|c|c|c|c|c|}
\hline & \multicolumn{3}{|c|}{ Descriptive statistics } & \multicolumn{6}{|c|}{ Unadjusted linear regression analyses } \\
\hline & \multirow{2}{*}{$\begin{array}{l}\text { ASz group } \\
(n=34) \\
\text { Mean (s.d.) }\end{array}$} & \multirow{2}{*}{$\begin{array}{l}\text { FHx group } \\
(n=24) \\
\text { Mean (s.d.) }\end{array}$} & \multirow{2}{*}{$\begin{array}{c}\text { TD group } \\
(n=42) \\
\text { Mean (s.d.) }\end{array}$} & \multicolumn{3}{|c|}{ ASz $v$. TD group, $P$} & \multicolumn{3}{|c|}{ FHX $v$. TD group, $P$} \\
\hline & & & & $d$ & $\beta(95 \% \mathrm{Cl})$ & $P$ & $d$ & $\beta(95 \% \mathrm{Cl})$ & $P$ \\
\hline \multicolumn{10}{|l|}{ Negative life events } \\
\hline $\begin{array}{l}\text { Total number } \\
\text { Distress at the time }\end{array}$ & $1.74(1.14)$ & $1.96(1.52)$ & $1.10(1.21)$ & 0.55 & $0.64(0.10$ to 1.18$)$ & 0.02 & 0.66 & $0.86(0.19$ to 1.54$)$ & 0.01 \\
\hline of the event & $1.44(1.00)$ & $1.51(1.12)$ & $1.26(1.18)$ & 0.16 & $0.18(-0.33$ to 0.69$)$ & 0.49 & 0.22 & $0.25(-0.34$ to 0.85$)$ & 0.39 \\
\hline Current distress & $0.88(0.88)$ & $0.91(0.95)$ & $0.58(0.73)$ & 0.37 & $0.30(-0.07$ to 0.66$)$ & 0.12 & 0.41 & $0.33(-0.08$ to 0.75$)$ & 0.12 \\
\hline \multicolumn{10}{|l|}{ Daily stressors } \\
\hline \multicolumn{10}{|c|}{ Frequency scores (score range) } \\
\hline Scholastic (0-24) & $12.09(3.37)$ & $12.04(4.12)$ & $11.00(3.84)$ & 0.30 & $1.09(-0.59$ to 2.76$)$ & 0.20 & 0.27 & $1.04(-0.98$ to 3.06$)$ & 0.31 \\
\hline Home $(0-12)$ & $4.76(2.39)$ & $4.92(2.08)$ & $3.76(2.28)$ & 0.44 & $1.00(-0.07$ to 2.07$)$ & 0.07 & 0.53 & $1.16(0.02$ to 2.29$)$ & 0.05 \\
\hline Peer (0-48) & $14.94(7.06)$ & $10.88(6.71)$ & $9.31(6.58)$ & 0.84 & $5.63(2.51$ to 8.76$)$ & 0.001 & 0.24 & $1.57(-1.82$ to 4.96$)$ & 0.36 \\
\hline Teacher (0-24) & $10.29(4.48)$ & $8.29(4.05)$ & $6.86(3.34)$ & 0.90 & $3.44(1.65$ to 5.23$)$ & $<0.001$ & 0.40 & $1.44(-0.41$ to 3.28$)$ & 0.13 \\
\hline \multicolumn{10}{|c|}{ Distress scores (score range) } \\
\hline Scholastic (0-3) & $1.43(0.49)$ & $1.17(0.57)$ & $1.07(0.65)$ & 0.63 & $0.37(0.10$ to 0.64$)$ & 0.008 & 0.16 & 0.10 ( -0.22 to 0.42$)$ & 0.54 \\
\hline Home $(0-3)$ & $1.10(0.61)$ & $1.11(0.66)$ & $0.76(0.68)$ & 0.53 & $0.34(0.04$ to 0.64$)$ & 0.03 & 0.53 & 0.35 (0.00 to 0.69$)$ & 0.05 \\
\hline Peer (0-3) & $1.15(0.67)$ & $0.80(0.71)$ & $0.81(0.57)$ & 0.56 & $0.34(0.06$ to 0.63$)$ & 0.02 & -0.00 & $-0.00(-0.32$ to 0.32$)$ & 0.99 \\
\hline Teacher (0-3) & $1.23(0.57)$ & $0.84(0.64)$ & $0.91(0.58)$ & 0.58 & $0.33(0.06$ to 0.59$)$ & 0.02 & -0.12 & $-0.07(-0.38$ to 0.23$)$ & 0.64 \\
\hline
\end{tabular}

( $\rho=0.50, P=0.003$ ); there were no other statistically significant correlations. Psychotic-like experiences were also strongly correlated with total daily stressor frequency scores in the $\mathrm{FHx}$ group ( $\rho=0.55, P=0.006$ ), but only a moderate correlation was observed in the TD group $(\rho=0.33, P=0.04)$. In the FHx group, total daily stressor frequency scores were also correlated positively with child-reported internalising $(\rho=0.49, P=0.02)$ and externalising ( $\rho=0.48, P=0.02)$ symptoms. Similarly, strong correlations were observed in the TD group between total daily stressor frequency scores and both child-reported internalising $(\rho=0.68, \quad P<0.001)$ and externalising $(\rho=0.61, \quad P<0.001)$ symptoms, and total daily stressor distress scores were significantly correlated with child-reported internalising symptoms $(\rho=0.65$, $P<0.001$ ). None of the symptom measures were associated with negative life events (neither exposure nor distress) in the $\mathrm{FHx}$ and TD groups $(P>0.05)$.

After excluding the five children meeting both ASz and FHx criteria, results were unchanged except that the relationship between daily stressor frequency scores and externalising symptoms in the FHx group was slightly reduced in magnitude ( $\rho=0.42, P=0.08$ ). In contrast, in the ASz group, the association between total daily stressor distress ratings and psychotic-like experiences increased in strength $(\rho=0.42, P=0.03)$.

\section{Discussion}

This is the first study conducted in the UK to prospectively examine environmental stressors in children at elevated risk for developing schizophrenia and also the first to compare children with different vulnerability profiles for the disorder. Children with a family history of schizophrenia and children presenting multiple antecedents of schizophrenia were more frequently exposed to major negative life events and milder daily stressors/ hassles respectively when compared with their typically developing peers. Furthermore, children in both the FHx and ASz groups reported greater distress resulting from these experiences, which did not appear to be explained by current mood or distress relating to other psychopathology. In all analyses, the magnitude of group differences reduced after excluding five children who met both $\mathrm{ASz}$ and FHx criteria (i.e. had a family history of schizophrenia and with multiple antecedents of schizophrenia), perhaps because children who present both developmental and genetic risk factors experience greatest risk of developing schizophrenia. Exploratory correlational analyses indicated that daily stressors and, to a lesser degree, negative life events were associated with current psychopathology.

Several limitations should be noted. First, the study included a relatively small sample, although the number of children at elevated risk was sufficiently large for us to be able to distinguish between those meeting criteria for the $\mathrm{ASz}$ and FHx groups. The sample also underrepresented children with multiple antecedents of schizophrenia with emotional symptoms in the clinical range. Second, no corrections were applied to reduce the risk of type 1 errors associated with multiple testing. Finally, in common with previous studies of children and adolescents, ${ }^{7,28,29}$ environmental stressors were assessed via self-report only. These are balanced against several strengths of the work. None of the children had ever experienced a psychotic episode or received psychotropic medication, and, with the exception of a small number of children identified via relatives' medical records, all were recruited using community-based screening. Thus, our findings are not biased by diagnosis (i.e. psychotic disorder) or help-seeking behaviour.

Relative to children in the TD group, those in the FHx and $\mathrm{ASz}$ groups experienced a greater number of negative life events, although the magnitude of effect in the ASz group was somewhat attenuated after adjustment for demographic factors. A greater number of life events was similarly observed in a study of adolescents with schizotypal personality disorder, ${ }^{7}$ but not in two investigations ${ }^{30,31}$ of youth meeting ultra-high-risk criteria (either: (a) attenuated psychotic symptoms, (b) brief, limited intermittent psychotic symptoms, or (c) genetic risk plus functional decline ${ }^{12}$ ) or in a study of young adults with a family history of schizophrenia. ${ }^{32}$ These inconsistencies may relate to differences in the participants' age or the specific life events examined. In the current study, children with a family history of schizophrenia also reported that these events were more distressing currently. Youth at ultra-high risk are likewise more distressed by life events than healthy controls. ${ }^{31}$ The specific negative life events examined in the current study may be particularly pertinent to children with a family history of schizophrenia. For example, having a parent who is acutely unwell may create an environment where some of these events are more likely to occur. 
Children in the ASz group, but not those in the FHx group, were more frequently exposed to peer- and teacher-related daily stressors and were more distressed by these experiences. Consistent with the fact that eligibility for the ASz group was partially determined on the basis of symptom presentation, children in the ASz group continued to have higher scores on measures of internalising and externalising symptoms and psychotic-like experiences at the time of stressor assessment at age 11-14 years (Table 1). These symptoms may influence the way in which children presenting with multiple antecedents of schizophrenia interact with their environment, thereby increasing the likelihood that they will encounter such daily stressors. In contrast, two previous studies, one of youth at ultra-high risk ${ }^{31}$ and the other of adolescents with schizotypal personality disorder, ${ }^{7}$ did not observe a higher frequency of daily stressors in at-risk youth relative to healthy controls. However, both studies reported that the at-risk youth were more distressed by these experiences. A possible explanation for this discrepancy is that our measure of daily stressor frequency, in contrast to the measures used in other studies, indexed not only the number of stressors experienced but also how frequently they occurred.

\section{Exploratory correlation analyses}

We also explored the relationship between environmental stressors and current symptoms; however, these findings should be interpreted with caution as no corrections were applied for multiple testing. We found that total daily stressor frequency scores were correlated positively with psychotic-like experiences in the FHx, ASz and TD groups, which concurs with a study of adults with psychotic disorders and healthy controls. ${ }^{33}$ Similar to previous studies in children, ${ }^{29}$ we also observed significant correlations between daily stressor frequency scores and childreported internalising and externalising symptoms in the $\mathrm{FHx}$ and TD groups. We did not observe this relationship in the ASz group and this finding is consistent with a study of ultra-high-risk youth in which daily hassles were not correlated with anxiety or depression, ${ }^{34}$ and may reflect a 'ceiling effect' in measurement. For example, it is possible that internalising and externalising symptoms among children in the ASz group were sufficiently high so as to be unaffected by daily hassles.

\section{Potential mechanisms}

One potential mechanism by which environmental stressors might interact with biological vulnerability to give rise to psychosis is via the hypothalamic-pituitary-adrenal (HPA) axis, ${ }^{1}$ which is largely responsible for coordinating physiological responses to stress. Evidence of abnormal HPA axis function, including enlarged pituitary volume, elevated cortisol secretion and reduced hippocampal volume, has been reported in individuals with first-episode psychosis, ultra-high-risk youth and relatives of patients with psychosis. ${ }^{35}$ Relatively little is known about HPA axis function in children at elevated risk for schizophrenia, although two studies reported elevated salivary cortisol in adolescents with schizotypal personality disorder ${ }^{36}$ and reduced hippocampal volume in children with a family history of schizophrenia ${ }^{37}$ compared with healthy controls. Studies linking assessments of environmental stressors with biological indices of HPA axis function in children at elevated risk for schizophrenia are needed.

Although accumulated evidence indicates that early adverse events increase the risk of developing psychotic-like experiences, little is known about the psychological processes underlying this association. ${ }^{38}$ Using data from a large prospective cohort study, Fisher and colleagues recently demonstrated that the relationship between childhood exposure to harsh parenting, and, to a lesser extent, bullying and domestic violence, and psychotic-like experiences in adolescence was mediated by anxiety, depressive symptoms, external locus of control and low self-esteem. ${ }^{39}$ Thus, the association between environmental stressors and psychoticlike experiences observed in the current cross-sectional study may be driven, at least in part, by specific, potentially targetable cognitive and affective difficulties.

\section{Implications}

The current findings, which require replication in larger samples, provide preliminary evidence that underlying vulnerability to psychosis is associated with the extent to which children experience and react to environmental stressors. Relative to children in the TD group, those in the FHx group reported feeling significantly greater distress in relation to negative life events currently, but their reports of distress at the time of the event were not significantly higher. All three groups of children reported greater distress at the time of the event, with lower levels of distress reported currently. We speculate that the significantly higher current distress ratings in the FHx group relative to the TD group may imply that the FHx group used less adaptive coping mechanisms, causing their distress to persist at higher levels over time. If so, cognitive-behavioural-based interventions might help high-risk youth develop more adaptive appraisals of environmental stressors and thereby reduce their emotional reactivity to these experiences. ${ }^{40,41}$ Longitudinal follow-up of the cohort will determine whether exposure to environmental stressors predicts transition to illness among children at elevated risk of developing schizophrenia.

\footnotetext{
Alexis E. Cullen, MSC, Department of Forensic and Neurodevelopmental Sciences, Institute of Psychiatry, King's College London, UK; Helen L. Fisher, PhD, CPsychol, AFBPSS, MRC Social, Genetic \& Developmental Psychiatry Centre, Institute of Psychiatry, King's College London, UK; Ruth E. Roberts, MSc, Department of Forensic and Neurodevelopmental Sciences, Institute of Psychiatry, King's College London, UK; Carmine M. Pariante, MD, MRCPsych, PhD, Section of Stress, Psychiatry and Immunology \& Perinatal Psychiatry, Department of Psychological Medicine, Institute of Psychiatry, King's College London, UK; Kristin R. Laurens, PhD, Department of Forensic and Neurodevelopmental Sciences, Institute of Psychiatry, King's College London, UK, and Research Unit for Schizophrenia Epidemiology, School of Psychiatry, University of New South Wales, and Schizophrenia Research Institute, Sydney, Australia

Correspondence: Alexis E. Cullen, Department of Forensic and Neurodevelopmental Sciences (Box P023), Institute of Psychiatry, De Crespigny Park, London SE5 8AF, UK. Email: alexis.cullen@kcl.ac.uk

First received 17 Feb 2013, final revision 21 Nov 2013, accepted 28 Nov 2013
}

\section{Funding}

This work was supported by funding to K.R.L. from a National Institute for Health Research (NIHR) Career Development Fellowship (CDF/08/01/015); a Bial Foundation Research Grant (35/06); a NARSAD Young Investigator Award (2005); and the British Medical Association Margaret Temple Award for schizophrenia research (2006). H.L.F. was supported by a Medical Research Council (MRC) Population Health Scientist award (G1002366).

\section{Acknowledgements}

The authors thank the children and caregivers who participated in the study, and the researchers and students who contributed to data collection. All authors are affiliated with the NIHR Specialist Biomedical Research Centre (BRC) for Mental Health at the South London and Maudsley National Health Service Foundation Trust and Institute of Psychiatry, King's College London, United Kingdom.

\section{References}

1 Walker E, Mittal V, Tessner K. Stress and the hypothalamic pituitary adrenal axis in the developmental course of schizophrenia. Annu Rev Clin Psychol 2008; 4: 189-216. 
2 Myin-Germeys I, van Os J. Stress-reactivity in psychosis: evidence for an affective pathway to psychosis. Clin Psychol Rev 2007; 27: 409-24.

3 Matheson SL, Shepherd AM, Pinchbeck RM, Laurens KR, Carr VJ. Childhood adversity in schizophrenia: a systematic meta-analysis. Psychol Med 2013; 43: $225-38$.

4 Morgan C, Fisher H. Environmental factors in schizophrenia: childhood trauma - a critical review. Schizophr Bull 2007; 33: 3-10.

5 Kelleher I, Harley M, Murtagh A, Cannon M. Are screening instruments valid for psychotic-like experiences? A validation study of screening questions for psychotic-like experiences using in-depth clinical interview. Schizophr Bull 2011; 37: 362-9.

6 Kelleher I, Harley M, Lynch F, Arseneault L, Fitzpatrick C, Cannon M. Associations between childhood trauma, bullying and psychotic symptoms among a school-based adolescent sample. Br J Psychiatry 2008; 193: 378-82.

7 Tessner KD, Mittal V, Walker EF. Longitudinal study of stressful life events and daily stressors among adolescents at high risk for psychotic disorders. Schizophr Bull 2011; 37: 432-41.

8 Laurens KR, Hodgins S, Maughan B, Murray RM, Rutter ML, Taylor EA. Community screening for psychotic-like experiences and other putative antecedents of schizophrenia in children aged 9-12 years. Schizophr Res 2007: 90: 130-46.

9 Laurens KR, Hodgins S, Taylor E, Murray RM. IS earlier intervention for schizophrenia possible?: Identifying antecedents of schizophrenia in children aged 9-12 years. In Schizophrenia: The Final Frontier (eds AS David, P McGuffin, S Kapur): 19-32. Psychology Press, 2011.

10 Gottesman II, Shields J. Schizophrenia: An the Epigenetic Puzzle. Cambridge University Press, 1982

11 Mortensen PB, Pedersen MG, Pedersen CB. Psychiatric family history and schizophrenia risk in Denmark: which mental disorders are relevant? Psychol Med 2010; 40: 201-10

12 Yung AR, McGorry PD. The prodromal phase of first-episode psychosis: past and current conceptualizations. Schizophr Bull 1996; 22: 353-70.

13 Laurens KR, Hodgins S, Mould GL, West SA, Schoenberg PL, Murray RM, et al. Error-related processing dysfunction in children aged 9 to 12 years presenting putative antecedents of schizophrenia. Biol Psychiatry 2010; 67: 238-45.

14 Macmanus D, Laurens KR, Walker EF, Brasfield JL, Riaz M, Hodgins S Movement abnormalities and psychotic-like experiences in childhood: markers of developing schizophrenia? Psychol Med 2012; 42: 99-109.

15 Cullen AE, De Brito SA, Gregory SL, Murray RM, Williams SC, Hodgins S, et al. Temporal lobe volume abnormalities precede the prodrome: a study of children presenting antecedents of schizophrenia. Schizophr Bull 2013; 39: 1318-27.

16 Cullen AE, Dickson H, West SA, Morris RG, Mould GL, Hodgins S, et al. Neurocognitive performance in children aged 9-12 years who present putative antecedents of schizophrenia. Schizophr Res 2010; 121: 15-23.

17 Matheson SL, Vijayan H, Dickson H, Shepherd AM, Carr VJ, Laurens KR. Systematic meta-analysis of childhood social withdrawal in schizophrenia and comparison with data from at-risk children aged 9-14 years. J Psychiat Res 2013; 47: 1061-8.

18 Dickson H, Calkins ME, Kohler CG, Hodgins S, Laurens KR. Misperceptions of facial emotions among youth aged 9-14 years who present multiple antecedents of schizophrenia. Schizophr Bull 2013; Jan 31 (Epub ahead of print).

19 Laurens KR, West SA, Murray RM, Hodgins S. Psychotic-like experiences and other antecedents of schizophrenia in children aged 9-12 years: a comparison of ethnic and migrant groups in the United Kingdom. Psychol Med 2008; 38: 1103-11.

20 Goodman R. Psychometric properties of the strengths and difficulties questionnaire. J Am Acad Child Adolesc Psychiatry 2001; 40: 1337-45.
21 Maxwell ME. Family Interview for Genetic Studies. National Institute of Mental Health, 1992

22 Office for National Statistics. Neighbourhood Statistics by Local Authority. National Statistics, 2010.

23 Heubeck B, O'Sullivan C. An exploration into the nature, frequency and impact of school hassles in the middle school years. Aust Psychol 1998; 33: 130-7.

24 Achenbach TM, Rescorla LA. Manual for the ASEBA Preschool Forms and Profiles. University of Vermont, Research Center for Children, Youth \& Families, 2001.

25 Laurens KR, Hobbs MJ, Sutherland M, Green MJ, Mould GL. Psychotic-like experiences in a community sample of 8,000 children aged 9-11 years: an item response theory analysis. Psychol Med 2012; 47: 1495-506.

26 Costello A, Edelbrock C, Kalas R, Kessler M, Klaric S. NIMH Diagnostic Interview Schedule for Children: Child Version. National Institute of Mental Health, 1982

27 Lipsey MW, Wilson DB. Practical Meta-Analysis. Sage Publications, 2001.

28 Barrett S, Heubeck B. Relationships between school hassles and uplifts and anxiety and conduct problems in grades 3 and 4. J Appl Dev Psychol 2000; 21: $537-54$

29 Kliewer W, Kung E. Family moderators of the relation between hassles and behavior problems in inner-city youth. J Clin Child Psychol 1998; 27: 278-92.

30 Devylder JE, Ben-David S, Schobel SA, Kimhy D, Malaspina D, Corcoran CM Temporal association of stress sensitivity and symptoms in individuals at clinical high risk for psychosis. Psychol Med 2013; 43: 259-68.

31 Phillips LJ, Edwards J, McMurray N, Francey S. Comparison of experiences of stress and coping between young people at risk of psychosis and a nonclinical cohort. Behav Cogn Psychother 2012; 40: 69-88.

32 Miller $\mathrm{P}$, Lawrie SM, Hodges A, Clafferty R, Cosway R, Johnstone EC. Genetic liability, illicit drug use, life stress and psychotic symptoms: preliminary findings from the Edinburgh study of people at high risk for schizophrenia Soc Psychiatry Psychiatr Epidemiol 2001; 36: 338-42.

33 Goldstone E, Farhall J, Ong B. Life hassles, experiential avoidance and distressing delusional experiences. Behav Res Ther 2011; 49: 260-6.

34 Thompson KN, Phillips LJ, Komesaroff P, Yuen HP, Wood SJ, Pantelis C, et al. Stress and HPA-axis functioning in young people at ultra high risk for psychosis. J Psychiatr Res 2007; 41: 561-9.

35 Aiello G, Horowitz M, Hepgul N, Pariante CM, Mondelli V. Stress abnormalities in individuals at risk for psychosis: a review of studies in subjects with familial risk or with "at risk" mental state. Psychoneuroendocrinology 2012; 37: 1600-13.

36 Mittal VA, Dhruv S, Tessner KD, Walder DJ, Walker EF. The relations among putative biorisk markers in schizotypal adolescents: minor physical anomalies, movement abnormalities, and salivary cortisol. Biol Psychiatry 2007; 61: 1179-86.

37 Sismanlar SG, Anik Y, Coskun A, Agaoglu B, Karakaya I, Yavuz Cl. The volumetric differences of the fronto-temporal region in young offspring of schizophrenic patients. Eur Child Adolesc Psychiatry 2010; 19: 151-7.

38 Schafer I, Fisher HL. Childhood trauma and psychosis - what is the evidence? Dialogues Clin Neurosci 2011; 13: 360-5.

39 Fisher HL, Schreier A, Zammit S, Maughan B, Munafo MR, Lewis G, et al. Pathways between childhood victimization and psychosis-like symptoms in the ALSPAC birth cohort. Schizophr Bull 2013; 39: 1045-55.

40 Maddox L, Jolley S, Laurens KR, Hirsch C, Hodgins S, Browning S, et al. Cognitive behavioural therapy for unusual experiences in children: a case series. Behav Cogn Psychother 2013; 41; 344-58.

41 Palmier-Claus JE, Dunn G, Lewis SW. Emotional and symptomatic reactivity to stress in individuals at ultra-high risk of developing psychosis. Psychol Med 2012; 42: 1003-12. 